\title{
Portable UWB RADAR Sensing System for Transforming Subtle Chest Movement Into Actionable Micro-Doppler Signatures to Extract Respiratory Rate Exploiting ResNet Algorithm
}

\author{
Umer Saeed ${ }^{\circledR}$, Syed Yaseen Shah, Abdullah Alhumaidi Alotaibi, Turke Althobaiti ${ }^{\circledR}$, \\ Naeem Ramzan ${ }^{\circledR}$, Senior Member, IEEE, Qammer H. Abbasi ${ }^{\circledR}$, Senior Member, IEEE, \\ and Syed Aziz Shah
}

\begin{abstract}
Contactless or non-invasive technology for the monitoring of anomalies in an inconspicuous and distant environment has immense significance in health-related applications, in particular COVID-19 symptoms detection, diagnosis, and monitoring. Contactless methods are crucial specifically during the COVID-19 epidemic as they require the least amount of involvement from infected individuals as well as healthcare personnel. According to recent medical research studies regarding coronavirus, individuals infected with novel COVID-19-Delta variant undergo elevated respiratory rates due to extensive infection in the lungs. This appalling sit-

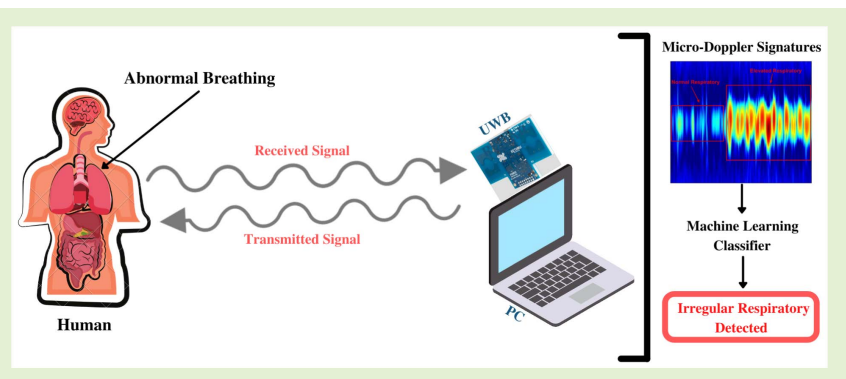
uation demands constant real-time monitoring of respiratory patterns, which can help in avoiding any pernicious circumstances. In this paper, an Ultra-Wideband RADAR sensor "XeThru X4M200" is exploited to capture vital respiratory patterns. In the low and high frequency band, X4M200 operates within the $6.0-8.5 \mathrm{GHz}$ and $7.25-10.20 \mathrm{GHz}$ band, respectively. The experimentation is conducted on six distinct individuals to replicate a realistic scenario of irregular respiratory rates. The data is obtained in the form of spectrograms by carrying out normal (eupnea) and abnormal (tachypnea) respiratory. The collected spectrogram data is trained, validated, and tested using a cutting-edge deep learning technique called Residual Neural Network or ResNet. The trained ResNet model's performance is assessed using the confusion matrix, precision, recall, F1-score, and classification accuracy. The unordinary skip connection process of the deep ResNet algorithm significantly reduces the underfitting and overfitting problem, resulting in a classification accuracy rate of up to $90 \%$.
\end{abstract}

Index Terms-COVID-19, UWB RADAR sensor, contactless healthcare, respiratory monitoring, deep learning, ResNet.

\section{INTRODUCTION}

C ORONAVIRUS is a broad family of viruses that can infect individuals and spread among humans in a variety

Manuscript received August 26, 2021; revised August 28, 2021; accepted September 2, 2021. Date of publication September 3, 2021; date of current version October 18, 2021. This work was supported in part by the Engineering and Physical Sciences Research Council (EPSRC) under Grant EP/R511705/1 and EP/T021063/1, in part by the Ajman University Internal Research Grant, and in part by Taif University, Taif, Saudi Arabia, through the Taif University Research Grant under Project TURSP-2020/277. The associate editor coordinating the review of this article and approving it for publication was Prof. Yongqiang Zhao. (Corresponding author: Umer Saeed.)

This work involved human subjects or animals in its research. Approval of all ethical and experimental procedures and protocols was granted by the College of Science and Engineering Ethics Committee under Application No. 300190109.

Please see the Acknowledgment section of this article for the author affiliations.

Digital Object Identifier 10.1109/JSEN.2021.3110367 of ways, including MERS-CoV, SARS-CoV, and the novel SARS-CoV-2 (COVID-19) [1]. COVID-19 infections have an extensive clinical spectrum, from asymptomatic contagion to mild upper respiratory tract sickness to acute viral pneumonia with respiratory collapse and even mortality [2]-[4]. Pulmonary function testing is a means to quantify COVID-19 impacts such as respiratory rate. The association between the respiratory pattern and COVID-19 symptoms is a known issue right now. The rate of respiration is the number of breaths per minute that a person takes while at rest [5]. Counting the number of breaths for one minute depending on how many times the chest rises is all that is required for respiratory rate. Fever, asthma, and other medical conditions can also cause respiratory rate to rise [6]. In COVID-19 instances, the respiratory rate is critical for determining the patients' pulmonary activity since aberrant values might suggest patient worsening [7], [8]. 
Measuring respiratory rate normally needs the assistance of a health professional, hence it is normally done at a hospital. The respiratory rate and pulmonary function study of the identified patients, however, raises the danger of contagiousness due to the clinical urgency produced by COVID-19 [9]. Because the majority of patients do not show signs of pulmonary distress at first, healthcare practitioners must make the tough decision to send them home and hope that they will be able to self-monitor. Some individuals with a moderate clinical presentation may deteriorate in the second week of sickness, according to the Centers for Disease Control and Prevention (CDC) [10], [11]. Patients in self isolation whose pulmonary functions and respiratory rate are unaffected and do not require hospitalisation should be followed utilising telemedicine technology [12], [13]. The significant risk of diagnosed individuals developing severe respiratory distress necessitates real-time respiratory rate monitoring of these individuals [14]. The Food and Drug Administration (FDA) has even approved the use of gadgets that can remotely monitor a patient's vital signs. However, there are few instruments available for real-time monitoring at home and the majority of them involve intrusive technologies such as cameras or wearable devices, for instance, watches, cuffs, belts, etc [15]-[17].

Certain smart contactless technologies have been proposed in the past to eliminate the inconvenience caused by wearables and other invasive gadgets to monitor crucial signs [18]-[20]. Continuous monitoring, even at night, is one of the benefits of contactless sensing [21]-[24]. During sleep, when wearable gadgets might be a distraction for the patients, they do not need to be aware of the contactless devices. Force sensor [25], multi-channel infrared sensor arrays [26], load cells [27], vibration sensor [28], pressure sensor [29], and Radio-Frequency (RF) are the most common contactless monitoring devices [30]. One of the most intriguing technologies among all is RF, which takes use of the electromagnetic waves that can be retrieved using RADAR technology. However, none of the existing RADAR-based techniques for aberrant respiratory rate estimation contains a comprehensive framework for analysing the patients in real-time and transferring the data to a healthcare practitioner for rapid action.

In this paper, we look at the prospect of adopting contactless (non-invasive/non-contact) technology to monitor real-time respiratory in COVID-19 patients. We present a system for monitoring COVID-19 patients that utilises off-the-shelf UltraWideband (UWB) RADAR sensor (XeThru X4M200 Respiration Sensor) created by NOVELDA [31]. The details about RADAR are provided in Section III and a complete framework of the proposed scheme is presented in Figure 1. This type of RADAR sensor is capable to monitor several diseases' symptoms through recognition of abnormal respiratory rates such as apnea, dyspnea, hyperpnea, tachypnea, hypopnea, bradypnea, orthopnea, platypnea, biot, cheyne-stokes, and kussmaul. However, we have focused primarily on tachypnea in this study. The normal and abnormal types of respiratory discussed in this paper are defined as follows:

1) Eupnea: Eupnea is the normal/regular respiratory pattern caused by healthy living and nutritious diet. Eupnea includes a respiratory rate of $12-20$ breaths per minute in general.

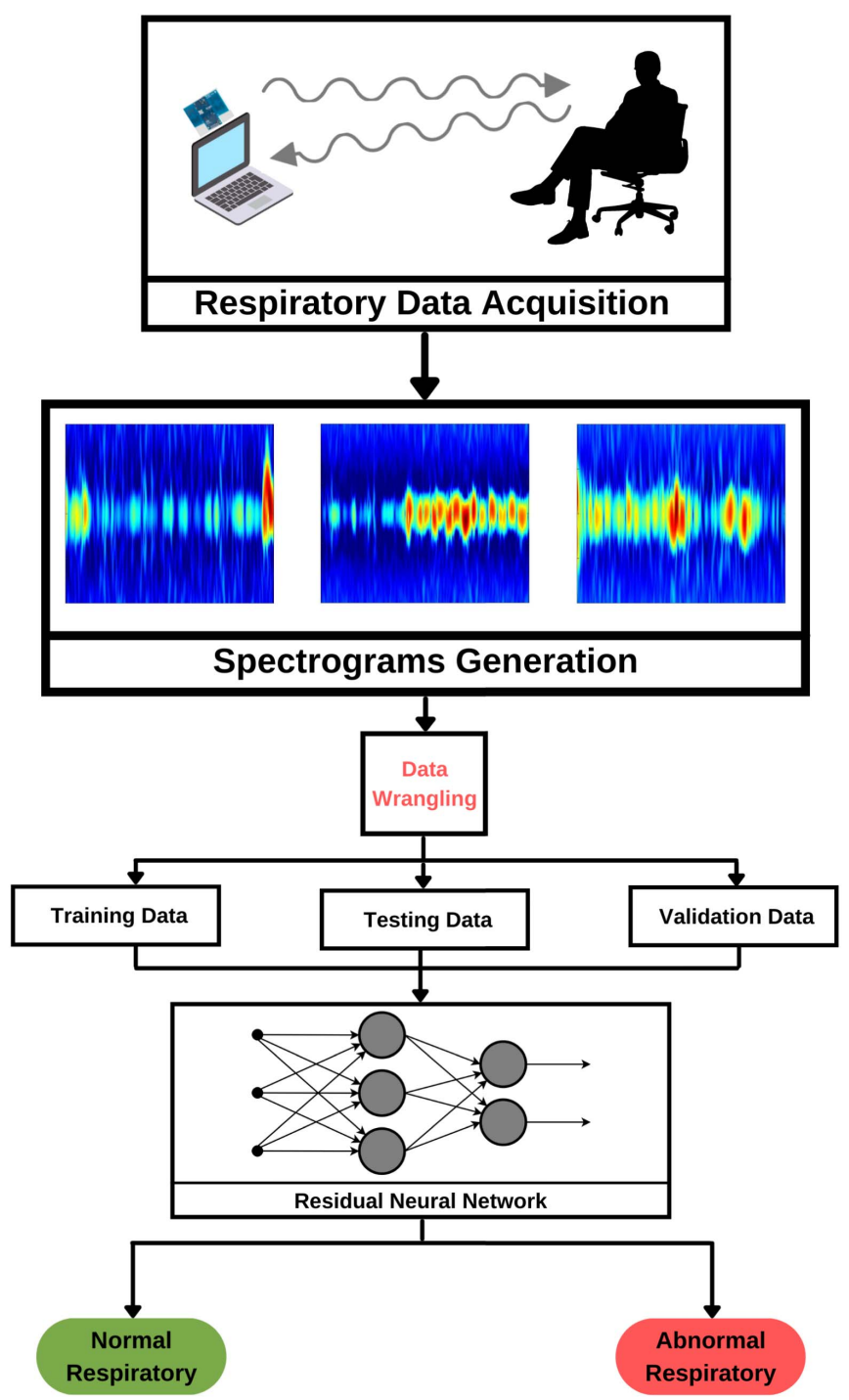

Fig. 1. Framework of the proposed scheme.

The pattern, rhythm, and depth of eupnea is regular as the wave form of this respiratory go up and down at normal rate.

2) Tachypnea: Tachypnea is an abnormal/irregular respiratory pattern, which is usually greater than 20 breaths per minute. In general, tachypnea is caused by pain, fever, hypoxia, or central nervous system issue. However, recently due to COVID-19-Delta variant, it has been noticed that patients may highly suffer from tachypnea and eventually lungs failure. Therefore, the timely monitoring and detection of patients' abnormal respiratory is of extreme significance, especially in the time of COVID-19.

\section{Related Work}

Acute Respiratory Distress Syndrome (ARDS) accounted for 10-15 percent of ICU admissions and 5 percent of general hospitalisations prior to the effects of the COVID-19 pandemic. ARDS is a kind of respiratory collapse due to extensive infection in the lungs that develops quickly. Symptoms comprise bluish skin coloration (cyanosis), fast breathing 
(tachypnea), and shortness of breath (dyspnea). During the early phases of the COVID-19 pandemic, data on the physiopathology of the infections emerged from asymptomatic, badly affected individuals, and even deceased persons. According to the available evidence, the lung damage is associated with a distinctive pulmonary vascular dysfunction in the early stages of infection [32], [33].

Further, this section provides information on cuttingedge work in the area of human action recognition, where RADAR-based sensing technologies were effectively employed in combination with intelligent machine learning and deep learning algorithms. In [34], to classify the data, the researchers employed Support Vector Machine (SVM), K-Nearest Neighbors (KNN), and Google-Net algorithm. The accuracy provided by these algorithms was 78.25 percent for SVM, 77.15 percent for KNN, and 74.70 percent for GoogleNet. Authors in [35] incorporated RADAR spectrograms of actions including walking, sitting, falling, and leaning down. For data preprocessing, spectrograms were employed classification task and images were transformed to greyscale. On the preprocessed data, the SVM and Deep Neural Network (DNN) algorithms were used. SVM attained an accuracy score of 78 percent, whereas DNN reported 87 percent accuracy.

Spectrograms obtained through RADAR technology were utilised in [36] for images classification and the SVM technique was then used to classify. For feature selection, the authors devised a sequential forward selection technique. Depending on the number of instances employed, the classification results were accurate up to 95 percent. In [37], deep learning techniques such as Recurrent Neural Network (RNN) with Long Short-Term Memory (LSTM) and Convolutional Neural Network (CNN) were used to identify six distinct human activities using Doppler images acquired through a RADAR. The activities include clapping, boxing, waving, walking, piaffe, and running. As per the findings of this paper, the RNN algorithm with LSTM obtained an accuracy rate of up to 92 percent, while the $\mathrm{CNN}$ reached an accuracy rate of up to 82 percent.

The authors of [38] integrated range maps and spectrograms of distinct human actions for classification purposes. This research article explored five different human actions: falling, sitting, bending, walking, and kneeling. Using KNN and Principal Component Analysis (PCA) algorithm, the simulations yielded an accuracy rate of up to 82 percent. Using the fusion technique, the authors in [39] were able to enhance the accuracy from 82 to 91 percent for diverse human actions using KNN and PCA. In [40], authors employed Impulse Radio Ultra-Wideband (IRUWB) RADAR to record twelve different types of human movements. The features in the spectrograms were defined using KNN algorithm in the data processing stage. The Doppler shifts and power spectrum were then extracted and classified using a CNN. The detection of human movements was obtained with up to 98 percent accuracy. The proposed scheme in [41] utilised IRUWB RADAR with CNN to identify daily living activities and falling. This research employed a binary classification technique to distinguish between the fall and any other form of activity in

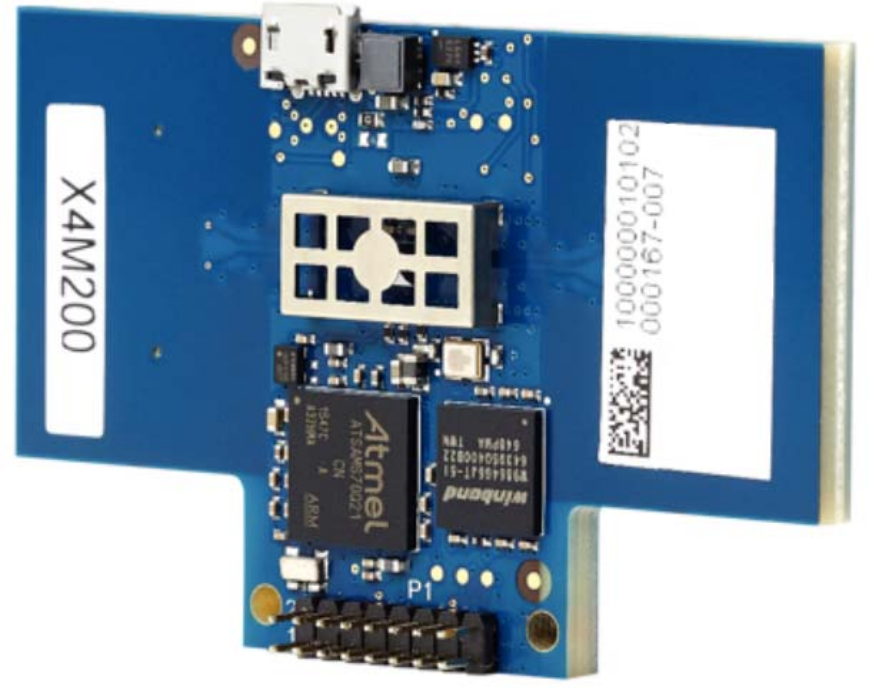

Fig. 2. XeThru X4M200 UWB RADAR sensor.

residence. The CNN technique was able to achieve up to 96 percent accuracy rate.

In [42], the authors used UWB RADAR to construct a dataset with ten participants ranging in age from 22-39 years, undertaking 15 distinct activities. The data were gathered while other people were still active inside the building. This was done to mimic a real-life care home setting where other residents live in nearby apartments. Using the random forest approach, the experiment obtained an overall accuracy of 80 percent. The authors in [43] employed a UWB RADAR to observe 7 people performing 4 different activities: standing, sitting, falling, and walking. With a result of 94 percent accuracy, the obtained data were processed through 10-fold cross-validation technique and it was discovered that KNN performed better. In [44], authors acquired data for binary classification of non-falling and falling incidents using UWB RADAR. Ten volunteers were used to collect data in three distinct areas within the proposed residence. To test their findings, the authors intentionally left one subject out and discovered that utilising a deep learning architecture CNNLSTM, the proposed scheme was able to reach an accuracy rate of up to 90 percent.

\section{Proposed Scheme}

\section{A. UWB RADAR Sensor}

In this study, UWB RADAR sensor (XeThru X4M200) is employed in order to develop a system, which can efficiently monitor irregular respiratory in COVID-19 patients or individuals suffering from ARDS. The UWB sensor is basically a respiration sensor based on RADAR technology consisting of built-in transmitter and receiver antennas. It is an industrialised sensor that meets international norms and is ready to be integrated into a product. The UWB sensor, which is in accordance with Novelda's proprietary X4 system-on-chip (SoC) with high integration, delivers very precise measurements of individuals respiratory rate as well as distance and movement details. This type of sensor is capable to monitor respiratory rate up to 


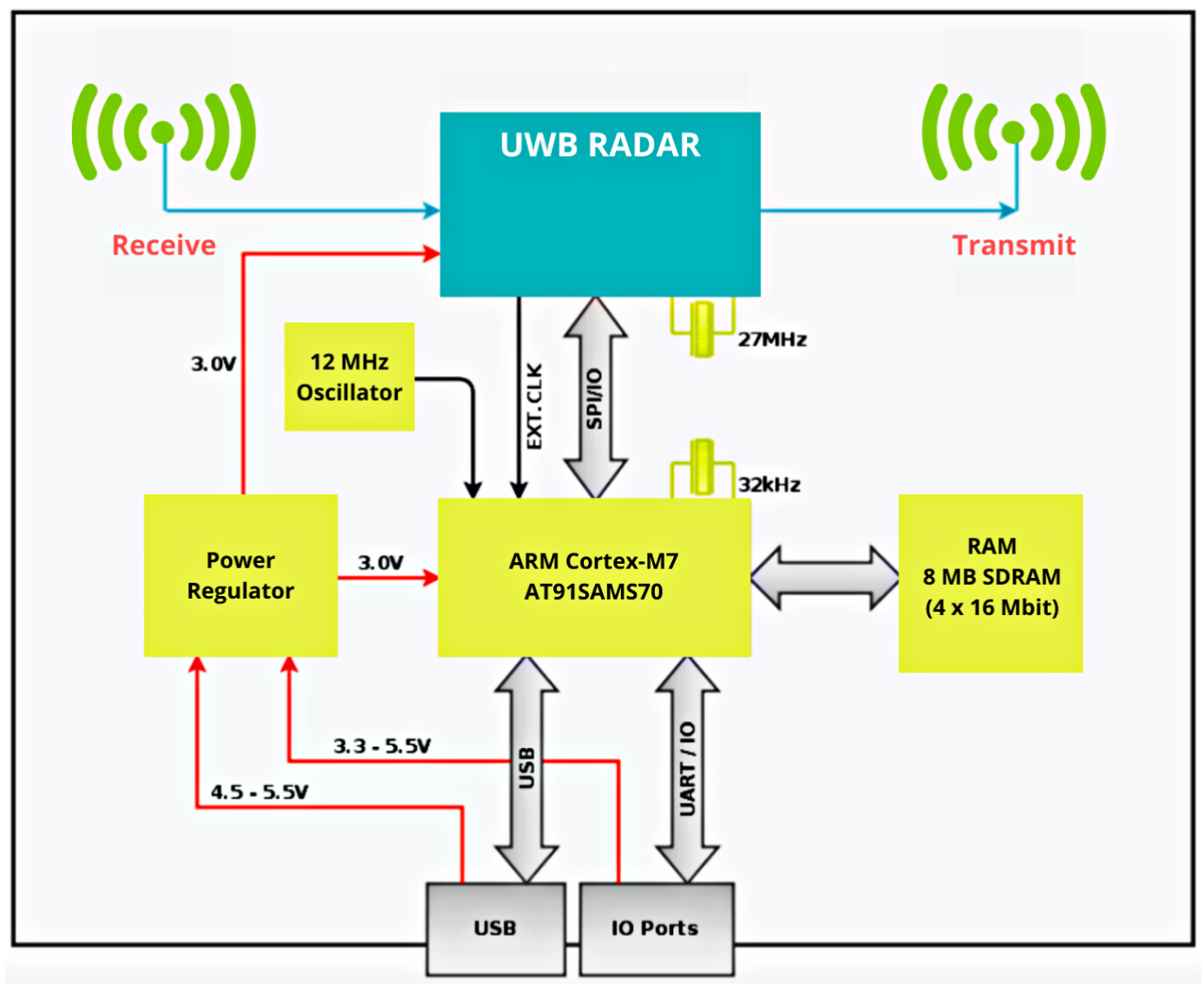

Fig. 3. Block diagram of UWB X4M200 RADAR sensor.

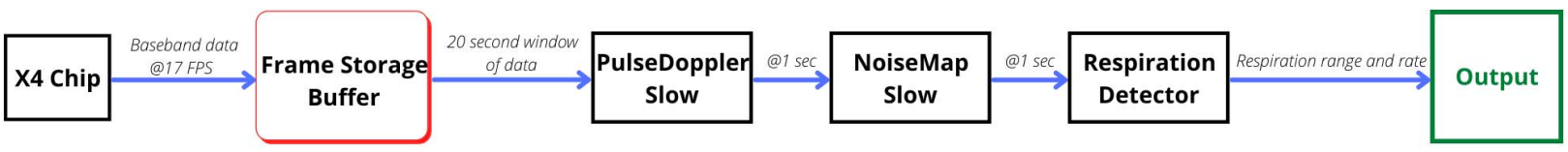

Fig. 4. Signal processing flow of UWB X4M200 RADAR sensor for respiratory detection.

5 meters when individual is still. The UWB RADAR sensor is revealed in Figure 2 and its complete block diagram is exhibited in Figure 3 - https://novelda.com.

\section{B. RADAR Signal Processing}

The UWB X4M200 sensor runs all firmware algorithms for motion detection and respiratory measures. The X4 UWB RADAR SoC can output 17 baseband data frames per second, as shown in the steps of Figure 4. A buffer is used to hold the RADAR data frames. Simultaneously, two Range-Doppler (RD) matrices are operating. Data from distinct times of RADAR frames is used in the fast and slow RD matrices. The lengths of the fast and slow phases vary by profile. Individual noise maps are used in both RD matrices to detect if a reflection at a specific distance and frequency exceeds a threshold. Enabling a noise map will provide multiple threshold values at various frequencies and distances. The noise maps will adjust to changes in the environment unless the noise map adaptation setting is off. Noise map adaptation is a continuous process that gradually eliminates the presence detection of reflectors with little movement at a certain distance. The noise map will not adjust, if a motionless individual is identified with a respiratory frequency matching the specified RPM range.

When an individual approaches the detection region, the fast RD matrix with its fast motion detector will identify the presence rapidly. Motion or motionless are the two states of the fast motion detector. These states are used by the fast $\mathrm{M} / \mathrm{N}$ connector to determine the Local-State-Fast. An M/N connector decides for the output to change, $\mathrm{M}$ out of $\mathrm{N}$ detections must have a specific value. All detection algorithms are executed once every second, which means that all outputs such as RPM, distance, state, and so on are updated once every second. When the target individuals are motionless, the slow RD matrix's slow motion detector and respiratory detector will identify their existence and estimate their respiratory rate and distance to the breathing target. Motion, motionless, and respiration are the three states of the respiratory detector and slow motion detector. These states are used by the slow $\mathrm{M} / \mathrm{N}$ connector 


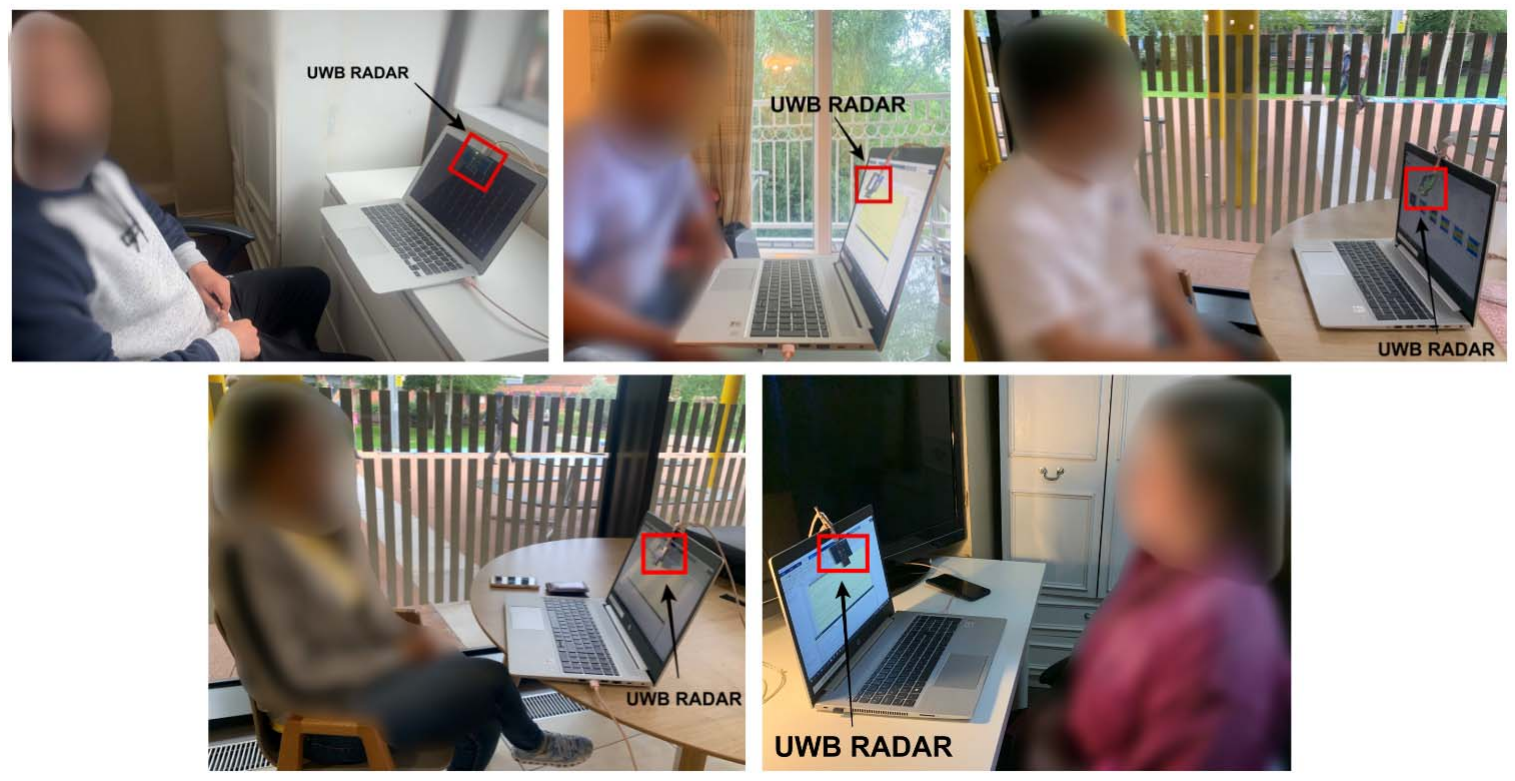

Fig. 5. Respiratory data acquisition in a static environment through diverse individuals.

to calculate the Local-State-Slow. An M/N connector decides for the output to change, $\mathrm{M}$ out of $\mathrm{N}$ detections must have a certain value.

\section{RADAR Micro-Doppler Signature}

The periodic motion of any structural element of an entity produces Micro-Doppler (MD) [45]. Micro-motion is created by the periodic motion, which causes side-bands around a bulk Doppler frequency. Consequently, the phase of such an object's RADAR return signal will change, for instance, human walking, human standing, or human chest movement. As a result, if the RADAR is coherent, variations in the phase values of successive pulses in pulsed RADAR or successive chirps in Frequency-Modulated Continuous-Wave (FMCW) RADAR would immediately correlate to changes in Doppler. To observe and evaluate the MD features, a velocity time spectrogram or the RD intrigue could be generated based on the data. The 2D Fast Fourier Transform (FFT) is used based on the data in FMCW RADAR to get Doppler information [46]. At first, every chirp is subjected to an FFT, which yields the range profile. Then, a second FFT is conducted on a certain number of successive chirps for a specific range bin. In general, Short-Time Fourier Transform (STFT) is employed to create these charts because, dissimilar to Fourier Transform, it gives temporal as well as frequency details [47]. This is accomplished by segmentation of data and applying the Fourier Transform of the segment in sequence. The temporal and frequency resolution are simultaneously affected by changing the window length, for instance, one increases when the other decreases. In RADAR data, the amount of Doppler detail is determined by the sampling capabilities of the hardware. In FMCW RADAR, the highest unequivocal Doppler frequency is $f_{d, \text { max }}=1 / 2 t_{s}$, where $t_{s}$ is the chirp time.
In this work, we consider a human respiratory monitoring scenario where a targeted point such as chest is located at a distance $D(t)$. The point of target movement in front of the RADAR is determined as $V(t)$, and the transmitted signal is represented as $S(t)$,

$$
S(t)=A \cos (2 \pi f t)
$$

The received signal is provided by $R(t)$,

$$
\mathrm{R}(\mathrm{t})=\mathrm{A}^{\prime} \cos \left(2 \pi f\left(\mathrm{t}-2 \frac{\mathrm{D}(\mathrm{t})}{c}\right)\right)
$$

$A$ ' is the reflection coefficient and $c$ is the speed of light. If the signal is reflected off a target point at an angle $\theta$ to the direction of RADAR, then the reflected signal can be expressed as,

$$
R(t)=A^{\prime} \cos \left(2 \pi f\left(1+\frac{2 V(t)}{c}\right) t-\frac{4 \pi D(\theta)}{c}\right)
$$

The Doppler shift that corresponds to it can be written as,

$$
f_{d}=f \frac{2 V(t)}{c}
$$

When dispersion of the entire human body is taken into account, the signal return becomes a composite of various moving parts such as hands, torso, arms, and head. Each component has its own acceleration and velocity of motion. Let $j$ be the various moving components of the body, then received signal can be written as

$$
R(t)=\sum_{j=0}^{N} A_{j} \cos \left(2 \pi f\left(1+\frac{2 V_{j}(t)}{c}\right) t-\frac{4 \pi D_{j}(0)}{c}\right)
$$

Consequently, the Doppler shift is the result of a complicated interaction of several Doppler shifts caused by the motion of distinct human body parts. Detection and diagnosis of human respiratory in a reliable fashion clearly depends upon the characteristics of the Doppler signatures. 
TABLE

PARAMETERS USED TO TRAIN THE DeEp ResNet Algorithm

\begin{tabular}{|l|l|}
\hline Algorithm & Parameters \\
\hline Residual Neural Network & $\begin{array}{l}\text { epochs }=50 \\
\text { activation = relu } \\
\text { optimizer = adam } \\
\text { loss = categorical-crossentropy }\end{array}$ \\
\hline
\end{tabular}

\section{Deep ResNet for Respiratory Classification}

Machine learning-based approaches have previously proved effective in a variety of applications [48]-[51]. In this article, we utilised a deep learning-based method known as Residual Neural Network or ResNet to classify normal and abnormal human respiratory using the acquired spectrograms. The skip connection technique is used to train such a network. The input used to feed a layer is also used to feed the output of a layer higher up the stack. The goal of training a ResNet is to get it to model the target function $f(x)$. If the network's output and input are linked, such as by establishing a skip connection, the network is strained to model $h(x)=f(x)-x$ rather than $f(x)$. This is stated as "Residual Learning" [52].

When a typical DNN is first initialised, the weights are almost zero, thus the network only outputs values that are close to zero. When a skip connection is introduced to the resultant network, it outputs a duplicate of its input, or to put it another way, it first models the identity function. If the objective function is near to the identity function, as is often the case, the training process can be greatly expedited. Furthermore, even though many layers in the network have yet to learn, the network can begin to improve by adding a high number of skip connections. Due to the skip connection approach, the signal can efficiently traverse the entire network. In other words, the ResNet can be regarded as a stack of residual units, all of which are a diminutive neural network with a procedure of skip connections. In this study, the hyperparameters used to train a ResNet algorithm in order to classify respiratory spectrograms are provided in Table I.

\section{Experimental Results and Analysis}

\section{A. Data Acquisition}

For this research study, the dataset was acquired using off-the-shelf UWB RADAR sensor. The details about the RADAR sensor are provided in Section III. To obtain distinct respiratory information, six human subjects were asked to sit on a chair in front of the UWB RADAR sensor at a distance of approximately 1 meter, nevertheless XeThru X4M200 UWB RADAR sensor has the ability to capture vital signs up to 5 meters. The UWB RADAR sensor was placed on top of the laptop at a fixed position, as shown in Figure 5. The experiments were conducted in a controlled environment. Moreover, the details about the subjects who participated in the experiment are given in Table II.

As shown in Figure 6, the subject was asked to perform each respiratory pattern for 15 seconds, Doppler[Hz] on the y-axis and Time[s] on the $\mathrm{x}$-axis. In the case of normal respiratory, the subject was asked to perform regular breathing pattern for
TABLE II

SubJects DetaILs Who PARTICIPATED IN ACQUIRING RESPIRATORY DATA

\begin{tabular}{|l|l|l|l|l|}
\hline Gender & Age & $\begin{array}{l}\text { Weight } \\
(\mathbf{K g})\end{array}$ & $\begin{array}{l}\text { Height } \\
(\mathbf{c m})\end{array}$ & Physique Type \\
\hline Male & 25 & 61 & 168 & Ectomorph \\
\hline Male & 30 & 85 & 170 & Mesomorph \\
\hline Male & 25 & 60 & 165 & Ectomorph \\
\hline Female & 28 & 52 & 158 & Ectomorph \\
\hline Female & 29 & 55 & 163 & Ectomorph \\
\hline Female & 26 & 65 & 166 & Mesomorph \\
\hline
\end{tabular}

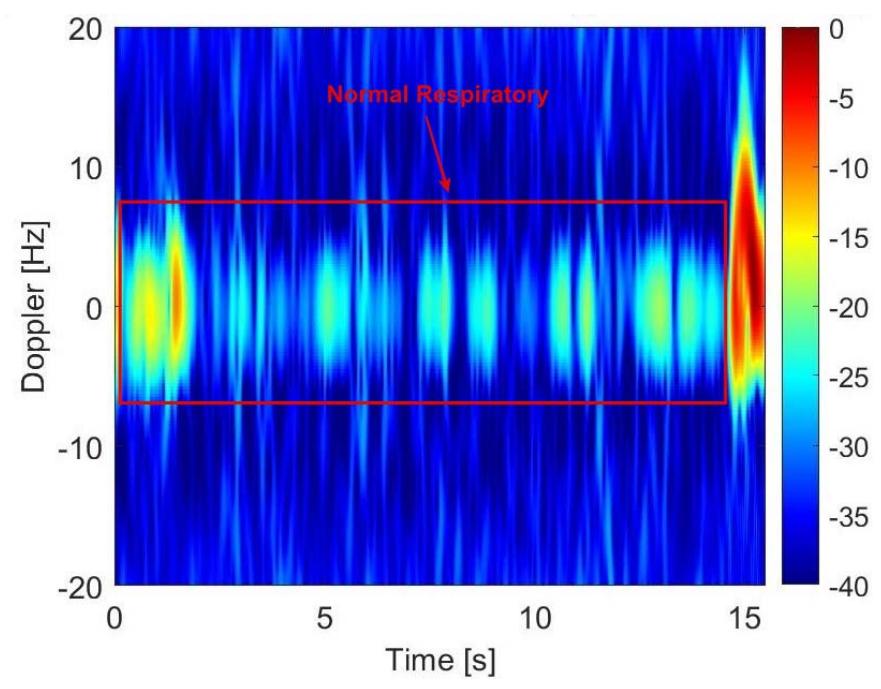

(a)

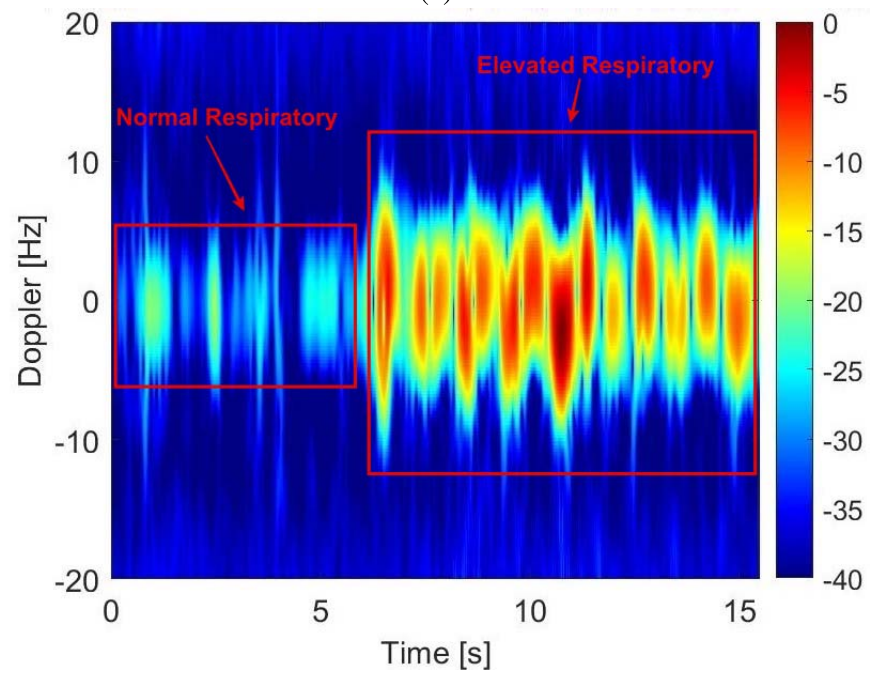

(b)

Fig. 6. Obtained spectrograms sample (a) normal/eupnea respiratory (b) abnormal/tachypnea respiratory.

straight 15 seconds. As can be seen in Figure 6(a), the normal respiratory lies between 8 to -8 in terms of Doppler[Hz]. In the case of abnormal respiratory, the subject was asked to perform a regular or normal breathing pattern for first 5 seconds (approximately) and an irregular or elevated breathing pattern for the rest of the 10 seconds. This was done in order to replicate a realistic scenario. The elevated (or rapid breathing) was performed by inhaling and exhaling air through nose 


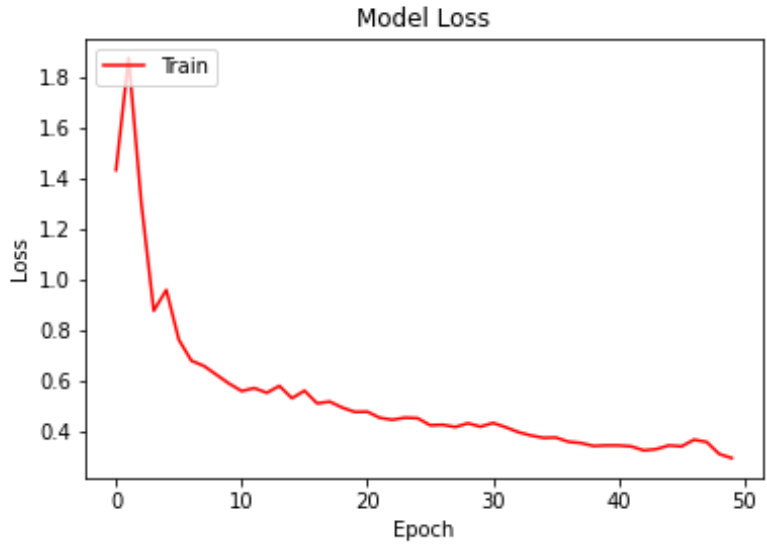

(a)

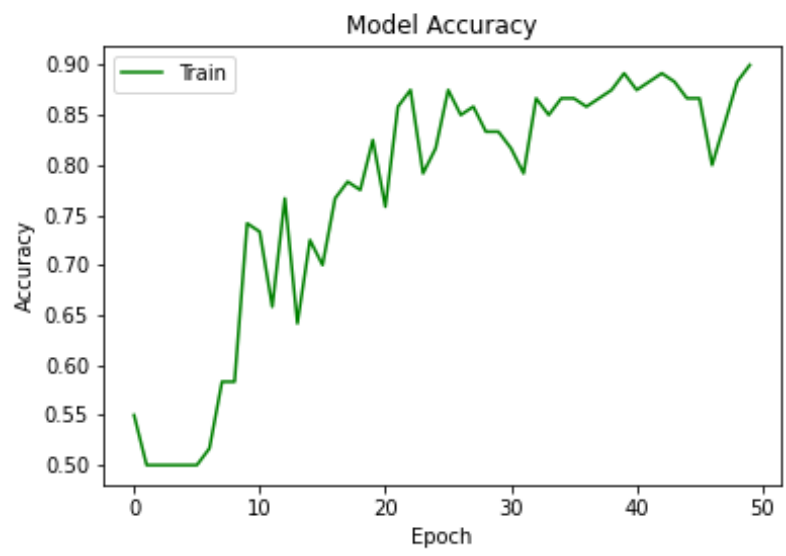

(b)

Fig. 7. The ResNet model (a) loss and (b) accuracy against distinct number of epochs.

fastly. As can be seen in Figure 6(b), the elevated respiratory has an immense impact on the acquired spectrograms as Doppler[Hz] shifts between 10 to -10 .

Every motion of the body generates a unique pattern on the spectrogram that can be used to discriminate between different types of human body motions. On the RADAR sensor, the RF signal is transmitted and received within the range when encountering any motion such as chest movement whilst breathing. The received signal on UWB RADAR sensor can be utilised to generate distinct spectrograms. In this work, each respiratory pattern were repeated several times by the subjects in order to record a dataset. A total of 230 spectrograms were generated for normal and abnormal respiratory, out of which 120 were used for training, 20 for validation, while 90 for testing purposes.

\section{B. Results and Discussion}

The ResNet approach used in this work to classify different human respiratory was written in Python with the TensorFlow and NumPy libraries. The confusion matrix, precision, recall, F1-score, and classification accuracy metrics were used to evaluate the performance of a trained model in this study (see Equation $6,7,8,9)$. The proportion of correctly recognised

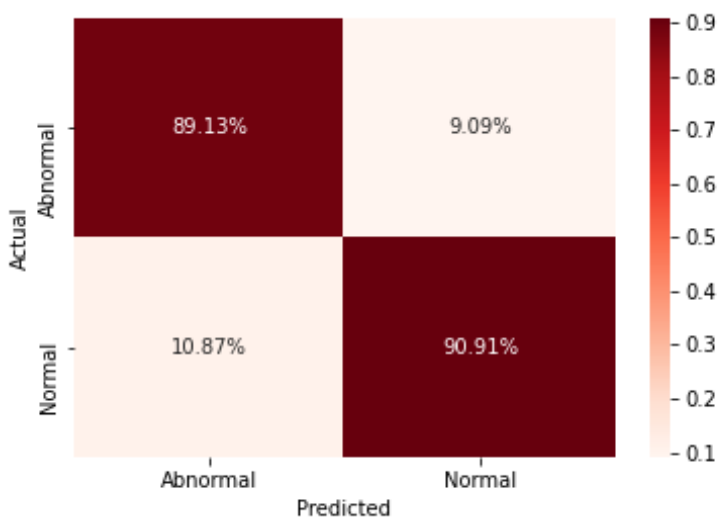

Fig. 8. Confusion matrix of normal and abnormal respiratory class through trained ResNet.

TABLE III

Classification Report of Resnet

\begin{tabular}{|l|l|l|l|}
\hline $\begin{array}{l}\text { Respiratory } \\
\text { Class }\end{array}$ & Precision & Recall & F1-score \\
\hline Normal & $91 \%$ & $89 \%$ & $90 \%$ \\
\hline Abnormal & $89 \%$ & $90 \%$ \\
\hline \hline \multicolumn{3}{|l|}{ Overall Accuracy: $90 \%$} \\
\hline
\end{tabular}

human respiratories to the total number of human respiratories can be described as classification accuracy. Moreover, F1-score or F-measure is the harmonic mean of recall and precision. F1score is an important metric since it gives a better measure of the incorrectly classified data samples than the classification accuracy metric.

$$
\begin{aligned}
\text { Precision } & =\frac{\text { True Positives }}{\text { Predicted Positives }} \\
\text { Recall } & =\frac{\text { True Positives }}{\text { Actual Positives }} \\
F 1-\text { score } & =2 \times\left(\frac{\text { Recall } \times \text { Precision }}{\text { Recall }+ \text { Precision }}\right) \\
\text { Accuracy } & =\frac{\text { Number of respiratories recognised }}{\text { Total number of respiratories }}
\end{aligned}
$$

The optimal parameters for training the ResNet model was achieved using the grid search approach. Taking size of the acquired dataset into consideration, the number of epochs were set to 50 while training the model. Once trained, the model performance was evaluated using distinct metrics. As revealed in Figure 7, the ResNet classifier was able to exceed an accuracy rate of 0.90 as the number of epochs increased, while the model loss was recorded less than 0.4. Moreover, Figure 8 shows a confusion matrix of normal and abnormal human respiratory classified by the trained deep ResNet algorithm. As can be seen, only few percent of misclassifications occurred between normal (eupnea) and abnormal (tachypnea) respiratory. Lastly, a complete classification report of the ResNet model in terms of percentage is exhibited in Table III. As can be noted, the normal respiratory class unveiled a precision rate of $91 \%$, recall $89 \%$, and F1-score $90 \%$. Whereas the abnormal respiratory class attained a precision rate of $89 \%$, recall $91 \%$, and F1-score $90 \%$. The deep ResNet model for 
both normal and abnormal respiratory classes procured an overall classification accuracy up to $90 \%$.

\section{CONCLUSION AND FutURE WORK}

A global epidemic COVID-19 is presently underway and the latest technology's role in aiding in this unwelcome scenario is significant. Abnormal respiratory is one of the most common symptoms of COVID-19 infection, especially among elderly people at the latter stages, nevertheless with the novel COVID-19-Delta variant, it is common to see more cases with abnormal respiratory problems amongst young adults. Therefore, a lightweight sophisticated system is required for real-time monitoring of the human respiratory. In this paper, a contactless or non-invasive approach is proposed based on an off-the-shelf UWB RADAR sensor merged with an intelligent deep neural network technique called ResNet. The proposed scheme is intended to detect and monitor abnormal respiratory patterns such as elevated breathing or tachypnea, which is common amidst coronavirus infection. Using the UWB RADAR sensor, the spectrograms of distinct human respiratory patterns were acquired and then used as image data to train, test, and validate the ResNet algorithm. Once trained, the ResNet model was put to the test by identifying between normal and abnormal human respiratory patterns. The simulation outcome revealed that ResNet obtained an overall accuracy of $90 \%$.

Certain limitations are related to this research study that we aspire to address in future research work. For instance, in a static and controlled setting, the proposed scheme can be employed for a specific subject at a time. Other than that, the investigations were not carried out on actual COVID19 infected individuals due to several complications. Consequently, future research work suggestions would be to include multiple individuals' respiratory patterns in a non-controlled environment with different limb motions. In addition, employing self-learning advanced machine learning algorithms and utilising the flexibility of the UWB RADAR-based platforms. Apart from that, other respiratory patterns including biot, bradypnea, sighing, and kussmaul will be investigated to improve the reliability of the proposed scheme.

\section{ACKNOWLEDGMENT}

Umer Saeed and Syed Aziz Shah are with the Research Centre for Intelligent Healthcare, Coventry University, Coventry CV1 5FB, U.K. (e-mail: saeedu3@uni.coventry.ac.uk; syed.shah@ coventry.ac.uk).

Syed Yaseen Shah is with the School of Computing, Engineering and Built Environment, Glasgow Caledonian University, Glasgow G4 0BA, U.K. (e-mail: syedyaseen.shah@gcu.ac.uk).

Abdullah Alhumaidi Alotaibi is with the Department of Science and Technology, College of Ranyah, Taif University, Taif 21944, Saudi Arabia (e-mail: a.alhumaidi@tu.edu.sa).

Turke Althobaiti is with the Faculty of Science, Northern Border University, Arar 91431, Saudi Arabia (e-mail: turke.althobaiti@nbu.edu.sa).

Naeem Ramzan is with the School of Engineering and Computing, University of the West of Scotland, Paisely PA1 2BE, U.K. (e-mail: naeem.ramzan@uws.ac.uk).

Qammer H. Abbasi is with the James Watt School of Engineering, University of Glasgow, Glasgow G12 8QQ, U.K. (e-mail: qammer.abbasi@ glasgow.ac.uk).

\section{REFERENCES}

[1] Coronavirus Disease 2019 (COVID-19): Situation Report, 82, World Health Org., Geneva, Switzerland, 2020.
[2] H. X. Bai et al., "Performance of radiologists in differentiating COVID-19 from non-COVID-19 viral pneumonia at chest CT," Radiology, vol. 296, no. 2, pp. E46-E54, Aug. 2020.

[3] J.-L. Vincent and F. S. Taccone, "Understanding pathways to death in patients with COVID-19," Lancet Respiratory Med., vol. 8, no. 5, pp. 430-432, May 2020.

[4] M. Rehman et al., "Contactless small-scale movement monitoring system using software defined radio for early diagnosis of COVID-19," IEEE Sensors J., vol. 21, no. 15, pp. 17180-17188, Aug. 2021.

[5] J. A. Hirsch and B. Bishop, "Respiratory sinus arrhythmia in humans: How breathing pattern modulates heart rate," Amer. J. Physiol., Heart Circulatory Physiol., vol. 241, no. 4, pp. H620-H629, Oct. 1981.

[6] D. L. Duncan, "The role of the nurse in supporting patients with asthma during the COVID-19 pandemic," Primary Health Care, vol. 30, no. 4, pp. 23-27, Jul. 2020.

[7] V. Polat and G. I. Bostanc1, "Sudden death due to acute pulmonary embolism in a young woman with COVID-19," J. Thrombosis Thrombolysis, vol. 50, no. 1, pp. 239-241, Jul. 2020.

[8] M. Vlachou et al., "Pulmonary thrombosis in COVID-19: Before, during and after hospital admission," J. Thrombosis Thrombolysis, vol. 51, no. 4, pp. 978-984, May 2021.

[9] B. Rai, A. Shukla, and L. K. Dwivedi, "Incubation period for COVID-19: A systematic review and meta-analysis," J. Public Health, vol. 221, pp. 1-8, Feb. 2021.

[10] Interim Clinical Guidance for Management of Patients With Confirmed Coronavirus Disease (COVID-19), Centers Disease Control Prevention, Atlanta, GA, USA, 2020.

[11] J. R. Hageman, "The coronavirus disease 2019 (COVID-19)," Pediatric Ann., vol. 49, no. 3, pp. e99-e100, 2020, doi: 10.3928/1938235920200219-01

[12] S. Bahl, R. P. Singh, M. Javaid, I. H. Khan, R. Vaishya, and R. Suman, "Telemedicine technologies for confronting COVID-19 pandemic: A review," J. Ind. Integr. Manage., vol. 5, no. 4, pp. 547-561, Dec. 2020.

[13] K. L. Rockwell and A. S. Gilroy, "Incorporating telemedicine as part of COVID-19 outbreak response systems," Amer. J. Manage. Care, vol. 26, no. 4, pp. 147-148, 2020.

[14] M. Rehman et al., "RF sensing based breathing patterns detection leveraging USRP devices," Sensors, vol. 21, no. 11, p. 3855 , 2021.

[15] R. Rogers, "Internet of Things-based smart healthcare systems, wireless connected devices, and body sensor networks in COVID-19 remote patient monitoring," Amer. J. Med. Res., vol. 8, no. 1, pp. 71-80, 2021.

[16] D. R. Seshadri et al., "Wearable sensors for COVID-19: A call to action to harness our digital infrastructure for remote patient monitoring and virtual assessments," Frontiers Digit. Health, vol. 2, p. 8, Jun. 2020.

[17] B. Dong et al., "Monitoring of atopic dermatitis using leaky coaxial cable," Healthcare Technol. Lett., vol. 4, no. 6, pp. 244-248, Dec. 2017.

[18] P. Leelaarporn et al., "Sensor-driven achieving of smart living: A review," IEEE Sensors J., vol. 21, no. 9, pp. 10369-10391, May 2021.

[19] X. Yang, D. Fan, A. Ren, N. Zhao, and M. Alam, "5G-based usercentric sensing at $C$-band," IEEE Trans. Ind. Informat., vol. 15, no. 5, pp. 3040-3047, May 2019.

[20] X. Yang et al., "Diagnosis of the hypopnea syndrome in the early stage," Neural Comput. Appl., vol. 32, no. 3, pp. 855-866, Feb. 2020.

[21] M. Piriyajitakonkij et al., "SleepPoseNet: Multi-view learning for sleep postural transition recognition using UWB," IEEE J. Biomed. Health Informat., vol. 25, no. 4, pp. 1305-1314, Apr. 2021.

[22] S. A. Shah, H. Abbas, M. A. Imran, and Q. H. Abbasi, "RF sensing for healthcare applications," in Backscattering and $R F$ Sensing for Future Wireless Communication. Hoboken, NJ, USA: Wiley, 2021, ch. 8, pp. 157-177. [Online]. Available: https://onlinelibrary.wiley.com/doi/abs/10.1002/9781119695721.ch8, doi: $10.1002 / 9781119695721 . c h 8$.

[23] D. Haider et al., "An efficient monitoring of eclamptic seizures in wireless sensors networks," Comput. Electr. Eng., vol. 75, pp. 16-30, May 2019.

[24] X. Yang et al., " $S$-band sensing-based motion assessment framework for cerebellar dysfunction patients," IEEE Sensors J., vol. 19, no. 19, pp. 8460-8467, Oct. 2019 .

[25] M. Cheng et al., "A review of flexible force sensors for human health monitoring," J. Adv. Res., vol. 26, pp. 53-68, Nov. 2020.

[26] X. Jiang, Y. Zhang, Q. Yang, B. Deng, and H. Wang, "Millimeter-wave array radar-based human gait recognition using multi-channel threedimensional convolutional neural network," Sensors, vol. 20, no. 19, p. 5466 , Sep. 2020 . 
[27] D. M. Minteer et al., "Pressure ulcer monitoring platform-A prospective, human subject clinical study to validate patient repositioning monitoring device to prevent pressure ulcers," Adv. Wound Care, vol. 9, no. 1, pp. 28-33, Jan. 2020.

[28] J. Huang et al., "Vibration monitoring based on flexible multi-walled carbon nanotube/polydimethylsiloxane film sensor and the application on motion signal acquisition," Nanotechnology, vol. 31, no. 33, Aug. 2020, Art. no. 335504.

[29] Y. Xiong et al., "A flexible, ultra-highly sensitive and stable capacitive pressure sensor with convex microarrays for motion and health monitoring," Nano Energy, vol. 70, Apr. 2020, Art. no. 104436.

[30] S. Z. Shah et al., "Privacy-preserving wandering behavior sensing in dementia patients using modified logistic and dynamic Newton Leipnik maps," IEEE Sensors J., vol. 21, no. 3, pp. 3669-3679, 2020.

[31] P. R. Bajurko and Y. Yashchyshyn, "Study of detection capability of Novelda impulse transceiver with external RF circuit," in Proc. IEEE 8th Int. Conf. Intell. Data Acquisition Adv. Comput. Syst., Technol. Appl. (IDAACS), vol. 2, Sep. 2015, pp. 693-696.

[32] C. I. Paules, H. D. Marston, and A. S. Fauci, "Coronavirus infectionsMore than just the common cold," J. Amer. Med. Assoc., vol. 323, no. 8, pp. 707-708, 2020.

[33] F. Potus et al., "Novel insights on the pulmonary vascular consequences of COVID-19," Amer. J. Physiol.-Lung Cellular Mol. Physiol., vol. 319, no. 2, pp. L277-L288, Aug. 2020.

[34] S. A. Shah and F. Fioranelli, "Human activity recognition : Preliminary results for dataset portability using FMCW radar," in Proc. Int. Radar Conf. (RADAR), Sep. 2019, pp. 1-4.

[35] B. Jokanovic, M. Amin, and F. Ahmad, "Radar fall motion detection using deep learning," in Proc. IEEE Radar Conf. (RadarConf), May 2016, pp. 1-6.

[36] B. Erol, M. G. Amin, B. Boashash, F. Ahmad, and Y. D. Zhang, "Wideband radar based fall motion detection for a generic elderly," in Proc. 50th Asilomar Conf. Signals, Syst. Comput., Nov. 2016, pp. 1768-1772.

[37] M. Wang, Y. D. Zhang, and G. Cui, "Human motion recognition exploiting radar with stacked recurrent neural network," Digit. Signal Process., vol. 87, pp. 125-131, Apr. 2019.

[38] B. Erol and M. Amin, "Generalized PCA fusion for improved radar human motion recognition," in Proc. IEEE Radar Conf. (RadarConf), Apr. 2019, pp. 1-5.

[39] W. Taylor, K. Dashtipour, S. A. Shah, A. Hussain, Q. H. Abbasi, and M. A. Imran, "Radar sensing for activity classification in elderly people exploiting micro-Doppler signatures using machine learning," Sensors, vol. 21 , no. 11 , p. 3881 , Jun. 2021.
[40] R. Qi, X. Li, Y. Zhang, and Y. Li, "Multi-classification algorithm for human motion recognition based on IR-UWB radar," IEEE Sensors J., vol. 20, no. 21, pp. 12848-12858, Nov. 2020.

[41] T. Han, W. Kang, and G. Choi, "IR-UWB sensor based fall detection method using CNN algorithm," Sensors, vol. 20, no. 20, p. 5948, Oct. 2020.

[42] K. Bouchard, J. Maitre, C. Bertuglia, and S. Gaboury, "Activity recognition in smart homes using UWB radars," Procedia Comput. Sci., vol. 170, pp. 10-17, Jan. 2020.

[43] A. Chowdhury, T. Das, S. Rani, A. Khasnobish, and T. Chakravarty, "Activity recognition using ultra wide band range-time scan," in Proc. 28th Eur. Signal Process. Conf. (EUSIPCO), Jan. 2021, pp. 1338-1342.

[44] J. Maitre, K. Bouchard, C. Bertuglia, and S. Gaboury, "Recognizing activities of daily living from UWB radars and deep learning," Expert Syst. Appl., vol. 164, Feb. 2021, Art. no. 113994.

[45] V. C. Chen, The Micro-Doppler Effect in Radar. Norwood, MA, USA: Artech House, 2019.

[46] V. Winkler, "Range Doppler detection for automotive FMCW radars," in Proc. Eur. Microw. Conf., 2007, pp. 166-169.

[47] D. P. Fairchild, R. M. Narayanan, E. R. Beckel, W. K. Luk, and G. A. Gaeta, "Through-the-wall micro-Doppler signatures," in Radar Micro-Doppler Signatures: Processing and Applications (Radar, Sonar and Navigation). Edison, NJ, USA: Institution of Engineering and Technology, 2014, pp. 97-137, doi: 10.1049/PBRA034E_ch5.

[48] U. Saeed, S. U. Jan, Y.-D. Lee, and I. Koo, "Fault diagnosis based on extremely randomized trees in wireless sensor networks," Rel. Eng. Syst. Saf., vol. 205, Jan. 2021, Art. no. 107284.

[49] S. U. Jan, Y. D. Lee, J. Shin, and I. Koo, "Sensor fault classification based on support vector machine and statistical time-domain features," IEEE Access, vol. 5, pp. 8682-8690, 2017.

[50] U. Saeed, Y. D. Lee, S. U. Jan, and I. Koo, "CAFD: Context-aware fault diagnostic scheme towards sensor faults utilizing machine learning," Sensors, vol. 21, no. 2, p. 617, 2021.

[51] Z. Ahmad, A. E. Prosvirin, J. Kim, and J.-M. Kim, "Multistage centrifugal pump fault diagnosis by selecting fault characteristic modes of vibration and using Pearson linear discriminant analysis," IEEE Access, vol. 8, pp. 223030-223040, 2020.

[52] K. He, X. Zhang, S. Ren, and J. Sun, "Deep residual learning for image recognition," in Proc. IEEE Conf. Comput. Vis. Pattern Recognit., Jun. 2016, pp. 770-778. 